\title{
CONVOLUTION ESTIMATES FOR SINGULAR MEASURES AND SOME GLOBAL NONLINEAR BRASCAMP-LIEB INEQUALITIES
}

\author{
HERBERT KOCH AND STEFAN STEINERBERGER
}

\begin{abstract}
We give a $L^{2} \times L^{2} \rightarrow L^{2}$ convolution estimate for singular measures supported on transversal hypersurfaces in $\mathbb{R}^{n}$, which improves earlier results of Bejenaru, Herr \& Tataru as well as Bejenaru \& Herr. The arising quantities are relevant in the study of the validity of bilinear estimates for dispersive partial differential equations. We also prove a class of global, nonlinear Brascamp-Lieb inequalities with explicit constants in the same spirit.
\end{abstract}

\section{INTRODUCTION}

1.1. Loomis-Whitney. The classical Loomis-Whitney inequality [6] bounds the $n$-dimensional volume of an open subset of $\mathbb{R}^{n}$ in terms of the size of its projections onto $(n-1)$-dimensions, one formulation is as follows: if the projections $\pi_{j}: \mathbb{R}^{n} \rightarrow \mathbb{R}^{n-1}$ are given by omitting the $j$-th component $\pi_{j}(x)=\left(x_{1}, \ldots, x_{j-1}, x_{j+1}, \ldots, x_{n}\right)$, then

$$
\int_{\mathbb{R}^{n}} f_{1}\left(\pi_{1}(x)\right) \ldots f_{n}\left(\pi_{n}(x)\right) d x \leq\left\|f_{1}\right\|_{L^{n-1}\left(\mathbb{R}^{n-1}\right)} \ldots\left\|f_{n}\right\|_{L^{n-1}\left(\mathbb{R}^{n-1}\right)}
$$

for all $f_{j} \in L^{n-1}\left(\mathbb{R}^{n-1}\right)$. The isoperimetric inequality is an immediate consequence: given a bounded domain $\Omega \subset \mathbb{R}^{n}$ and letting $f_{j}$ be the characteristic function of $\pi_{j}(\Omega)$, then any reasonable definition of surface measure satisfies

$$
\left\|f_{j}\right\|_{L^{n-1}\left(\mathbb{R}^{n}\right)}^{n-1} \leq|\partial \Omega|
$$

and thus the Loomis-Whitney inequality implies

$$
|\Omega|=\int_{\mathbb{R}^{n}} f_{1}\left(\pi_{1}(x)\right) \ldots f_{n}\left(\pi_{n}(x)\right) d x \leq\left\|f_{1}\right\|_{L^{n-1}\left(\mathbb{R}^{n-1}\right)} \ldots\left\|f_{n}\right\|_{L^{n-1}\left(\mathbb{R}^{n-1}\right)} \leq|\partial \Omega|^{\frac{n}{n-1}} .
$$

The proof of the Loomis-Whitney inequality combines an elementary combinatorial setup with induction on dimension, where each induction step is an application of Hölders inequality. A non-linear and local form of this result was given by Bennett, Carbery \& Wright [4]; if the $\pi_{j}$ are submersions with the kernels of $d \pi_{j}$ spanning the whole of $\mathbb{R}^{n}$, then the nonlinear Loomis-Whitney inequality still holds locally (with appropriate cut-off functions and constants).

1.2. Convolution inequalities. A variant in $\mathbb{R}^{3}$ arises in the Fourier analysis of nonlinear dispersive equations and is due to Bejenaru, Herr \& Tataru 2]. Let $\Sigma_{1}, \Sigma_{2}, \Sigma_{3} \subset \mathbb{R}^{3}$ be three surfaces with $C^{1+\alpha}$ regularity, bounded in diameter by 1 and uniformly transversal in the sense that for any three points $x_{i} \in \Sigma_{i}$ the associated normal vectors $\nu_{i}$ satisfy

$$
\left|\operatorname{det}\left(\nu_{1}, \nu_{2}, \nu_{3}\right)\right| \geq \frac{1}{2}
$$

Writing $\mu_{\Sigma_{i}}$ for the two-dimensional Hausdorff measure $\mathcal{H}^{2}$ restricted to $\Sigma_{i}$, we identify $f_{1} \in$ $L^{2}\left(\Sigma_{1}, \mu_{\Sigma_{1}}\right)$ with the distribution

$$
f_{1}(\psi)=\int_{\Sigma_{1}} f_{1}(x) \psi(x) d \mu_{\Sigma_{1}}(x) \quad \psi \in \mathcal{D}\left(\mathbb{R}^{3}\right)
$$

and analogously for $f_{2} \in L^{2}\left(\Sigma_{2}, \mu_{\Sigma_{2}}\right)$, which allows us to define the convolution as

$$
\left(f_{1} * f_{2}\right)(\psi)=\int_{\Sigma_{1}} \int_{\Sigma_{2}} f_{1}(x) f_{2}(y) \psi(x+y) d \mu_{\Sigma_{1}}(x) d \mu_{\Sigma_{2}}(y) .
$$


Here and below, we can always assume all arising functions to be nonnegative; if the inequalities hold for nonnegative functions, then they also hold for all measurable functions. Thickening the surfaces one sees by the coarea formula that

$$
\left(f_{1} * f_{2}\right)(z)=\int_{\Sigma_{1} \cap\left(z-\Sigma_{2}\right)} \gamma_{3}^{-1}(x, z-x) f_{1}(x) f_{2}(z-x) d \mathcal{H}^{1}
$$

where $\gamma_{3}(x, y)$ is the cosine of the angle between $\nu_{1}(x)$ and $\nu_{2}(y)$ for $x \in \Sigma_{1}$ and $y \in \Sigma_{2}$. Bejenaru, Herr \& Tataru then show that the restriction is well-defined in $L^{2}\left(\Sigma_{3}, d \mu_{\Sigma_{3}}\right)$ and that it satisfies

$$
\left\|f_{1} * f_{2}\right\|_{L^{2}\left(\Sigma_{3}\right)} \lesssim\left\|f_{1}\right\|_{L^{2}\left(\Sigma_{1}\right)}\left\|f_{2}\right\|_{L^{2}\left(\Sigma_{2}\right)} .
$$

The behavior under linear transformations yields additional information: one should be able to weaken the assumption on the transversality to $\left|\operatorname{det}\left(\nu_{1}, \nu_{2}, \nu_{3}\right)\right| \geq \gamma>0$ at the cost of increasing the implicit constant by a factor of order $\gamma^{-\frac{1}{2}}$ and, indeed, this is done in [2] at the cost of assuming certain conditions on diameter, Hölder exponent and Hölder norm. A dual formulation is achieved by introducing a weight function $f_{3} \in L^{2}\left(\Sigma_{3}\right)$ and rewriting

$$
\int_{\Sigma_{3}}\left(f_{1} * f_{2}\right)(x) f_{3}(-x) d x=\left(f_{1} * f_{2} * f_{3}\right)(0)
$$

Considering thickened surfaces $\Sigma_{i}^{*}=\Sigma_{i}+B(0, \varepsilon)$, where $B(0, \varepsilon)$ is the ball of radius $\varepsilon$ in $\mathbb{R}^{3}$, and assuming $f_{1} \in L^{2}\left(\Sigma_{1}^{*}\right), f_{2} \in L^{2}\left(\Sigma_{2}^{*}\right)$ and $f_{3} \in L^{2}\left(\Sigma_{3}^{*}\right)$, then ignoring all underlying geometry implies with Hölder that

$$
\begin{aligned}
\left|\left(f_{1} * f_{2} * f_{3}\right)(0)\right| & =\left|\int_{\mathbb{R}^{3}} \mathcal{F}\left(f_{1} * f_{2} * f_{3}\right)(x) d x\right| \\
& =\left|\int_{\mathbb{R}^{3}} \hat{f}_{1}(\xi) \hat{f}_{2}(\xi) \hat{f}_{3}(\xi) d \xi\right| \leq\left\|\hat{f}_{1}\right\|_{L^{3}}\left\|\hat{f}_{2}\right\|_{L^{3}}\left\|\hat{f}_{3}\right\|_{L^{3}}
\end{aligned}
$$

where $\mathcal{F}$ and ^ denote the Fourier transform. One way of looking at the Bejenaru-Herr-Tataru statement is that for $\varepsilon \rightarrow 0$ the transversal structure of the Fourier supports implies additional cancellation and allows us to conclude

$$
\int_{\mathbb{R}^{3}} \hat{f}_{1}(\xi) \hat{f}_{2}(\xi) \hat{f}_{3}(\xi) d x \lesssim\left\|\hat{f}_{1}\right\|_{L^{2}}\left\|\hat{f}_{2}\right\|_{L^{2}}\left\|\hat{f}_{3}\right\|_{L^{2}} .
$$

The proof given by Bejenaru, Herr \& Tataru is quite remarkable: it uses induction on scales à la Wolff and has inspired recent work by Bennett \& Bez 3 on Brascamp-Lieb inequalities. The work of Bennett \& Bez was then used by Bejenaru \& Herr [1] to extend [2] to arbitrary dimensions under the natural scaling condition of the codimensions adding up to the space dimension. All three papers treat the nonlinearity in a perturbative fashion.

1.3. Applicability. Results of this type are related to (multilinear) restriction problems and a bilinear estimates for partial differential equations of dispersive type, where the type of nontrivial interaction of two characteristic hypersurfaces $\Sigma_{1}+\Sigma_{2}$ with a third hypersurface $\Sigma_{3}$ determines whether a bilinear estimate is available. Bejenaru \& Herr 11, for example, use their generalized version of the Bejenaru-Herr-Tataru result to obtain locally well-posedness for the 3D Zakharov system in the full subcritical regime.

\section{Statement of Results}

2.1. The simplest case. We are interested in general convolution inequalities for curved submanifolds of $\mathbb{R}^{n}$. The simplest case, taken from [2, is given by three transversal hyperplanes in $\mathbb{R}^{3}$ equipped with the two-dimensional Hausdorff measure $\mathcal{H}^{2}$

$$
\begin{aligned}
& \Sigma_{1}=\left\{(x, y, z) \in \mathbb{R}^{3}: x=0\right\} \\
& \Sigma_{2}=\left\{(x, y, z) \in \mathbb{R}^{3}: y=0\right\} \\
& \Sigma_{3}=\left\{(x, y, z) \in \mathbb{R}^{3}: z=0\right\}
\end{aligned}
$$


and smooth functions $f \in L^{2}\left(\Sigma_{1}, \mathcal{H}^{2}\right), g \in L^{2}\left(\Sigma_{2}, \mathcal{H}^{2}\right)$ and $h \in L^{2}\left(\Sigma_{3}, \mathcal{H}^{2}\right)$. The convolution can be written down in an explicit fashion

$$
(f * g)(x, y, z):=\int f\left(y, z^{\prime}\right) g\left(x, z-z^{\prime}\right) d z^{\prime}
$$

and then duality yields that the estimate

$$
\|f * g\|_{L^{2}\left(\Sigma_{3}, \mathcal{H}^{2}\right)} \leq\|f\|_{L^{2}\left(\Sigma_{1}, \mathcal{H}^{2}\right)}\|g\|_{L^{2}\left(\Sigma_{2}, \mathcal{H}^{2}\right)}
$$

is equivalent to the estimate

$$
\left|\int f(y, z) g(x,-z) h(x, y) d x d y d z\right| \leq\|f\|_{L^{2}\left(\Sigma_{1}, \mathcal{H}^{2}\right)}\|g\|_{L^{2}\left(\Sigma_{2}, \mathcal{H}^{2}\right)}\|h\|_{L^{2}\left(\Sigma_{3}, \mathcal{H}^{2}\right)},
$$

which in itself is simply the three-dimensional Loomis-Whitney inequality. Note that the affine structure of the hyperplanes is crucial for the proof to work as it allows for an explicit parametrization of the integration fibers: in particular, this proof is not stable under small perturbations of the underlying surfaces.

2.2. Setup. Our general setup is as follows. Let $\Sigma_{i}(i=1,2,3)$ be $n_{i}$-dimensional Lipschitz manifolds in $\mathbb{R}^{n}$ with codimensions adding up to the space dimension, i.e.

$$
\sum_{i=1}^{3}\left(n-n_{i}\right)=n \quad \text { or } \quad n_{1}+n_{2}+n_{3}=2 n .
$$

This condition will be necessitated by scaling. Let $\mu_{\Sigma_{i}}$ be the $n_{i}$-dimensional Hausdorff measure restricted to $\Sigma_{i}$. We associate with every $f \in L_{l o c}^{1}\left(\Sigma_{i}, \mu_{i}\right)$ the signed measure $f \mu_{\Sigma_{i}}$. For every $i \in\{1,2,3\}$, there exists an orthonormal basis $\nu_{i, j}, 1 \leq j \leq n-n_{i}$, of normal vectors at every point of $\Sigma_{i}$ almost everywhere. Since $\left(n-n_{1}\right)+\left(n-n_{2}\right)+\left(n-n_{3}\right)=n$, this means that for every $(x, y, z) \in \Sigma_{1} \times \Sigma_{2} \times \Sigma_{3}$, we can define the matrix $N(x, y, z)$ by collecting the $n$ vectors $\nu_{i, j}$ as columns,

$$
N(x, y, z)=\left(\nu_{i . j}\right)
$$

The natural measure of transversality $\gamma$ is then defined by

$$
\Sigma_{1} \times \Sigma_{2} \times \Sigma_{3} \ni(x, y, z) \rightarrow \gamma(x, y, z)=|\operatorname{det}(N(x, y, z))| .
$$

We call the surfaces transversal if this local measure of transversality is uniformly bounded from below $\gamma \geq \gamma_{0}>0$ for all $(x, y, z) \in \Sigma_{1} \times \Sigma_{2} \times \Sigma_{3}$, whenever all three normal vectors are defined.

2.3. A convolution inequality. Our first result is a global version of [1] without the requirements on Hölder continuity, Hölder norms or bounds on the diameter - furthermore we are able to give an explicit constant.

Theorem 1 (Convolution inequality). Suppose that the $\Sigma_{i}$ are given as the graphs of Lipschitz functions and that

$$
\gamma(x, y, z) \geq \gamma_{0}
$$

Then, for all $f_{1} \in L^{2}\left(\Sigma_{1}\right), f_{2} \in L^{2}\left(\Sigma_{2}\right)$, we have

$$
\left\|f_{1} * f_{2}\right\|_{L^{2}\left(\Sigma_{3}\right)} \leq \gamma_{0}^{-\frac{3}{2}}\left\|f_{1}\right\|_{L^{2}\left(\Sigma_{1}\right)}\left\|f_{2}\right\|_{L^{2}\left(\Sigma_{2}\right)} .
$$

The constant does not have the form $\gamma_{0}^{-\frac{1}{2}}$ of the case of linear hypersurfaces; this is due to the fact that in the proof we are forced to use rough estimates to control a nonlocal interaction between locally defined linear maps - studying the factor explicitely can give slightly better bounds in many cases.

The following questions are natural but, to the best of our knowledge, open.

(1) Suppose that

$$
\inf \left\{\gamma(x, y, z): x \in \Sigma_{1}, y \in \Sigma_{2}, z \in \Sigma_{3}, x+y=z\right\} \geq \eta_{0}>0 .
$$

Is there a constant $C=C\left(\eta_{0}\right)$ so that the convolution estimate holds with this constant? We do not even know the answer if $\eta_{0}$ is larger than $1 / 2$. 
(2) Can one replace the factor $\gamma_{0}^{-3 / 2}$ by $C \gamma_{0}^{-1 / 2}$ ? Our proof shows this to be the case if the submanifolds are controlled Lipschitz perturbations of linear subspaces.

Our proof gives the following intermediate refinement of Theorem 1. If we assume that

$$
\inf \left\{\gamma(x, y, z): x, \tilde{x} \in \Sigma_{1}, y, \tilde{y} \in \Sigma_{2}, z \in \Sigma_{3}, x+\tilde{y}=\tilde{x}+y=z\right\}=: \gamma_{0}>0
$$

then

$$
\left\|f_{1} * f_{2}\right\|_{L^{2}\left(\Sigma_{3}\right)} \leq \gamma_{0}^{-\frac{3}{2}}\left\|f_{1}\right\|_{L^{2}\left(\Sigma_{1}\right)}\left\|f_{2}\right\|_{L^{2}\left(\Sigma_{2}\right)} .
$$

2.4. Nonlinear Brascamp-Lieb inequalities. Our approach to the convolution problem is flexible enough to allow us to deduce new global, nonlinear Brascamp-Lieb inequalities. We formulate the Brascamp-Lieb inequalities for the space $\mathbb{R}^{n}$ and three nonlinear mappings $\phi_{i}: \mathbb{R}^{n} \rightarrow \mathbb{R}^{n_{i}}$, where again

$$
n_{1}+n_{2}+n_{3}=2 n .
$$

Note that the problem now lies in the nonlinear fiber structure induced by preimages $\phi_{i}^{-1}$. We merely assume the $\phi_{i}$ to be $C^{1}$-submersions (i.e. $D \phi_{i}$ has rank $n_{i}$ ). Let $N_{i}(x)$ be the null space of $D \phi_{i}(x)$ of dimension $n-n_{i}$. We recall that

$$
\left(n-n_{1}\right)+\left(n-n_{2}\right)+\left(n-n_{3}\right)=n
$$

and assume that the nullspaces span $\mathbb{R}^{n}$ at every point. By an abuse of notation we identify $N_{i}$ with a matrix having an orthonormal basis as columns. We introduce a measure of transversality via

$$
\gamma_{0}=\inf _{z \in \mathbb{R}^{n_{3}}} \inf _{\left\{x, y: \phi_{3}(x)=\phi_{3}(y)=z\right\}} \sup _{O \in O(n): O N_{3}(x)=N_{3}(y)}\left|\operatorname{det}\left(O N_{1}(x), N_{2}(y), N_{3}(y)\right)\right| .
$$

This notion is inspired by the proof for the convolution, where such a definition is required in order for the Brascamp-Lieb case to behave in an analogous fashion as the convolution. To shorten the notation for the coarea formula we will henceforth write for a matrix $A$

$$
|A|=\left(\operatorname{det} A A^{T}\right)^{1 / 2}
$$

Furthermore, we will use $\sigma_{j}(x)$ to denote the singular values of $D \phi_{3}(x)$, i.e. the square roots of the eigenvalues of $D \phi_{3} D \phi_{3}^{T}$, ordered by their size, $\sigma_{1} \leq \sigma_{2} \leq \cdots \leq \sigma_{n_{3}}$. Using this notation, we have in particular that

$$
\left|D \phi_{3}(x)\right|=\prod_{j=1}^{n_{3}} \sigma_{j}
$$

and obtain for the operator norm of the linear mapping that

$$
\left\|D \phi_{3}(x)\right\|_{\ell^{2} \rightarrow \ell^{2}}=\sigma_{n_{3}} .
$$

Since the $D \phi_{i}$ are assumed to always have maximal rank, we necessarily have $\sigma_{1}>0$. Introducing additional notation, we set

$$
\rho_{1}(x)=\left|D \phi_{3}(x)\right|^{-\frac{n-n_{2}}{n_{3}}} \prod_{j=n-n_{2}+1}^{n_{3}} \sigma_{j}
$$

as well as

$$
\rho_{2}(y)=\left|D \phi_{3}(x)\right|^{-\frac{n-n_{1}}{n_{3}}} \prod_{j=n-n_{1}+1}^{n_{3}} \sigma_{j} .
$$

We observe that $n-n_{1}+n-n_{2}=n_{3}$. Let

$$
\rho=\sup _{z \in \mathbb{R}^{n_{3}}} \sup _{\left\{x, y: \phi_{3}(x)=\phi_{3}(y)=z\right\}} \rho_{1}(x) \rho_{2}(y) .
$$

Theorem 2 (Loomis-Whitney/Brascamp-Lieb). Under these assumptions, if

$$
\rho<\infty \text { and } \gamma_{0}>0
$$


then for all $f_{i} \in L^{2}\left(\mathbb{R}^{n_{i}}\right)$

$$
\int_{\mathbb{R}^{n}}\left(\prod_{i=1}^{3}\left|D \phi_{1}\right|\left|D \phi_{2} \| D \phi_{3}\right|\right)^{\frac{1}{2}} f_{1}\left(\phi_{1}(x)\right) f_{2}\left(\phi_{2}(x)\right) f_{3}\left(\phi_{3}(x)\right) d x \leq \sqrt{\frac{\rho}{\gamma_{0}}}\left\|f_{1}\right\|_{L^{2}}\left\|f_{2}\right\|_{L^{2}}\left\|f_{3}\right\|_{L^{2}} .
$$

The geometric weights arise as compensation of two possible kinds of symmetries: the geometric factor under the integral compensates for the possibility of coordinate changes in the image. We would like to obtain a formulation that is invariant under composing any of the three mappings $\phi_{i}$ with an invertible linear mapping $A: \mathbb{R}^{n_{i}} \rightarrow \mathbb{R}^{n_{i}}$, which introduces a Gramian determinant as Jacobian - this can happen in each image space independently which implies the necessity of the product structure of the weight. We do not quite achieve this desired invariance, since $\rho$ changes under diffeomorphisms of $\mathbb{R}^{n_{3}}$. This observation leads to a trivial improvement (minimizing over all possible diffeomorphisms) which we do not explicitely formulate. In addition the transversality of the kernels of $D \phi_{i}(x)$ has to enter the estimate. As for the convolution our condition is not entirely local and the question whether purely local transversality measures suffice, remains open.

\section{Proof of Linear Convolution inequalities}

3.1. Outline. The purpose of this section is to prove Theorem 1 in the case of the surfaces $\Sigma_{i}$ being linear subspaces and Theorem 2 for linear maps $\phi_{i}$. These special cases are known: it suffices to use the previously outlined approach via the Loomis-Whitney inequality and combine it with a change of variables. However, as outlined before, that proof is not stable. We will give a new proof of this special case; that proof will then be stable enough to handle the nonlinear setting as well. We start with a description of the underlying geometry, which translates into statements about parallelepipeds. Geometric properties about these parallelepipeds will determine factors coming from the transformation formula.

3.2. Basic facts about parallelepipeds. Let $H_{1}, H_{2}$ and $H_{3}$ be subspaces of $\mathbb{R}^{n}$ such that the direct sum of the orthogonal spaces yield $\mathbb{R}^{n}$, i.e.

$$
\mathbb{R}^{n}=H_{1}^{\perp} \oplus H_{2}^{\perp} \oplus H_{3}^{\perp} .
$$

Denote $n_{i}=\operatorname{dim}\left(H_{i}\right)$ and thus $\operatorname{codim}\left(H_{i}\right)=\operatorname{dim}\left(H_{i}^{\perp}\right)=n-n_{i}$. The vectors $\left(v_{j}^{i}\right)_{i=1,2,3, j=1, \ldots, n-n_{i}}$ are chosen such that $\left(v_{j}^{i}\right)_{j=1, \ldots, n-n_{i}}$ form an orthonormal basis of $H_{i}^{\perp}$. There is a unique dual basis $\left(w_{j}^{i}\right)_{i=1,2,3, j=1, \ldots, n-n_{i}}$ defined by the relations

$$
\left\langle v_{j}^{i}, w_{l}^{k}\right\rangle=\delta_{i k} \delta_{j l} .
$$

We write $V_{i}$ for the $n \times\left(n-n_{i}\right)$ matrix containing $\left(v_{j}^{i}\right)_{j=1, \ldots, n-n_{i}}$ and $W_{i}$ for the matrix of the same size containing $\left(w_{j}^{i}\right)_{j=1, \ldots, n-n_{i}}$. Our condition on the codimensions implies that concatenation yields square matrices

$$
V=\left(V_{1}, V_{2}, V_{3}\right) \quad \text { and } \quad W=\left(W_{1}, W_{2}, W_{3}\right)
$$

satisfying

From this, obviously

$$
V^{T} W=\operatorname{id}_{n \times n} .
$$

$$
\gamma:=|\operatorname{det}(V)|=|\operatorname{det} W|^{-1} .
$$

We recall that, given a matrix $A$, we use $|A|$ as an abbreviation for Gramian determinants, i.e.

$$
|A|=\left|\operatorname{det}\left(A A^{t}\right)\right|^{1 / 2},
$$

where $A$ may be any $n \times \cdot$ matrix and the number can be understood as the $\cdot$-dimensional Hausdorff measure of the parallelepiped formed by the vectors. In particular, in concordance with our previous use of the notation $\gamma_{3}$ in the fiber representation

$$
\gamma_{i}:=\left|W_{i}^{t}\right| .
$$

For simplicity we understand the indices as integers modulo 3 below. 
Lemma 1. For each $i \in\{1,2,3\}$, we have

$$
\left|\left(W_{i-1} W_{i+1}\right)^{t}\right|=\gamma^{-1}
$$

and

$$
\left|\left(V_{i-1} V_{i+1}\right)^{t}\right|=\gamma\left|W_{i}^{t}\right|
$$

Proof. We show a set inclusion about spaces spanned by these vectors:

$$
\operatorname{span}\left(W_{i}-V_{i}\right) \subset \operatorname{span}\left(W_{i-1} W_{i+1}\right) .
$$

Using that we have dual basis, a vector is in the right-hand set if and only if its projections onto any vector from $W_{i}$ is 0 , which is precisely the case if its projection onto any vector from $V_{i}$ is 0 . Now, with duality and orthonormality of elements in $V_{i}$

$$
\left\langle v_{i}^{a}, w_{i}^{b}-v_{i}^{b}\right\rangle=\left\langle v_{i}^{a}, w_{i}^{b}\right\rangle-\left\langle v_{i}^{a}, v_{i}^{b}\right\rangle=\delta_{a b}-\delta_{a b}=0 .
$$

Now, using the multilinearity of the determinant, we get

$$
\gamma^{-1}=\operatorname{det}\left(W_{i-1} W_{i+1} W_{i}\right)=\operatorname{det}\left(W_{i-1} W_{i+1} V_{i}\right) .
$$

Since we are dealing with dual bases, the volume of the $\mathrm{p}$ described by the determinant factors into

$$
\begin{aligned}
\gamma^{-1} & =\operatorname{det}\left(W_{i-1} W_{i+1} V_{i}\right) \\
& =\operatorname{det}\left(\left(W_{i-1} W_{i+1}\right)^{t}\left(W_{i-1} W_{i+1}\right)\right)^{1 / 2} \operatorname{det}\left(V_{i}^{t} V_{i}\right)^{1 / 2} \\
& =\left|\left(W_{i-1} W_{i+1}\right)^{t}\right| .
\end{aligned}
$$

A similar argument implies

$$
\operatorname{span}\left(W_{i}\left(W_{i} W_{i}^{t}\right)^{-1}-V_{i}\right) \subset \operatorname{span}\left(V_{i-1}, V_{i+1}\right)
$$

and hence

$$
\gamma=\operatorname{det}\left(V_{1} V_{2} V_{3}\right)=\operatorname{det}\left(V_{1} V_{2} W_{3}\left(W_{3} W_{3}^{t}\right)^{-1}\right)=\left|\left(V_{1} V_{2}\right)^{t}\right|\left|W_{3}^{t}\right|^{-1}
$$

which is the second identity.

3.3. Proof of the linear case. Using these geometric considerations, we are now able to produce a complete proof of the linear case. The main idea consists in applying Cauchy-Schwarz twice, once globally on a surface and once within the fiber of integration. This will lead to a suitable decoupling of the quantity allowing for a full solution via a further change of coordinates.

Proposition 1. Let $H_{1}, H_{2}$ and $H_{3}$ be as above. Then, for all $f_{1} \in L^{2}\left(H_{1}\right), f_{2} \in L^{2}\left(H_{2}\right)$,

$$
\left\|f_{1} * f_{2}\right\|_{L^{2}\left(H_{3}\right)} \leq \frac{1}{\sqrt{\gamma}}\left\|f_{1}\right\|_{L^{2}\left(H_{1}\right)}\left\|f_{2}\right\|_{L^{2}\left(H_{2}\right)} .
$$

Proof. Let $V_{i}$ and $V$ be as above and let $W_{i}$ be the dual basis to $V_{i}$. Then $H_{3}=W_{1} \times W_{2}$ and $\left|\left(W_{1}, W_{2}\right)^{t}\right|=\gamma$. Thus, with $m^{d}$ the $d$ dimensional Lebesgue measure, by the area formula and Lemma 1 .

$$
\int_{H^{3}}\left(f_{1} * f_{2}\right)^{2} d \mathcal{H}^{n_{3}}=\gamma^{-1} \int_{\mathbb{R}^{n_{3}}}\left(f_{1} * f_{2}\left(\left(W_{1}, W_{2}\right) y\right)^{2} d m^{n_{3}}(y) .\right.
$$

Moreover, with $s_{i} \in \operatorname{span}\left(W_{i}\right)$, by the coarea and area formulas

$$
\begin{aligned}
f_{1} * f_{2}\left(s_{1}+s_{2}+s_{3}\right) & =\left|\left(V_{1}, V_{2}\right)^{t}\right|^{-1} \int_{\text {span } W_{3}} f_{1}\left(s_{2}+t\right) f_{2}\left(s_{1}+t\right) d \mathcal{H}^{n-n_{3}}(t) \\
& =\frac{\left|W_{3}^{t}\right|}{\left|\left(V_{1}, V_{2}\right)^{t}\right|} \int_{\mathbb{R}^{n-n_{3}}} f_{1}\left(s_{2}+W_{3} z\right) f_{2}\left(s_{1}-W_{3} z\right) d m^{n-n_{3}}(z) \\
& =\gamma^{-1} \int_{\mathbb{R}^{n-n_{3}}} f_{1}\left(s_{2}+W_{3} z\right) f_{2}\left(s_{1}-W_{3} z\right) d m^{n-n_{3}}(z)
\end{aligned}
$$


where we used the second identity of Lemma 1. We continue

$$
\begin{aligned}
\left\|f_{1} * f_{2}\right\|_{L^{2}\left(H_{3}\right) \leq}^{2} \leq \gamma^{-3} & \int_{\mathbb{R}^{n_{3}}} \int_{\mathbb{R}^{n-n_{3}}} f_{1}^{2}\left(W_{2} y_{2}+W_{3} y_{3}\right) d m^{n-n_{3}}\left(y_{3}\right) \\
& \times \int_{\mathbb{R}^{n-n_{3}}} f_{2}^{2}\left(W_{1} y_{1}+W_{3} y_{3}\right) d m^{n-n_{3}}\left(y_{3}\right) d m^{n_{3}}\left(y_{1}, y_{2}\right) \\
= & \gamma^{-3}\left(\int_{\mathbb{R}^{n_{1}}} f_{1}^{2}\left(W_{2} y_{2}+W_{3} y_{3}\right) d m^{n_{1}}\left(y_{2}, y_{3}\right)\right) \\
& \times\left(\int_{\mathbb{R}^{n_{2}}} f_{2}^{2}\left(W_{1} y_{1}+W_{3} y_{3}\right) d m^{n_{2}}\left(y_{1}, y_{3}\right)\right) \\
= & \gamma^{-1}\left\|f_{1}\right\|_{L^{2}\left(H_{1}\right)}^{2}\left\|f_{2}\right\|_{L^{2}\left(H_{2}\right)}^{2}
\end{aligned}
$$

where we used again the considerations of (3.3).

\section{Convolution estimate: Proof of Theorem 1}

4.1. Outline. In this section we extend the previous argument from hyperplanes to general polyhedral surfaces. We emphasize that the problem has no special intrinsic connection to polyhedral surfaces and we use them solely out of convenience: they are well suited for approximating $C^{1}$-surfaces and, due to their piecewise linear nature, allow for a relatively slick reduction to the purely linear case as localizing will lead us with a locally linear geometry. Naturally, since we need to be able to carry out a limit process in the end, all our estimates will be independent of the number of faces of the polyhedral surfaces.

4.2. Fiber representations. There is an explicit expression for the convolution in terms of integration along the corresponding fiber, that will be useful in the proof of the statement. We will explicitely write $f_{i, \mu_{i}}$ to highlight the importance of the surface along with function value in the following impression. For a fixed $z \in \Sigma_{3}$, if

$$
\Gamma_{z}=\left\{x \in \Sigma_{1}: z-x \in \Sigma_{2}\right\}=\Sigma_{1} \cap\left(z-\Sigma_{2}\right),
$$

then, by thickening the surfaces and using the coarea formula,

$$
\left(f_{1, \mu_{1}} * f_{2, \mu_{2}}\right)(z)=\int_{x \in \Gamma_{z}} \gamma_{3}^{-1}(x, y) f_{1}(x) f_{2}(z-x) d \mathcal{H}^{n-n_{3}}(x)
$$

where the Gramian determinant $\gamma_{3}$ is given by

$$
\gamma_{3}(x, y)=\left|\operatorname{det}\left(\left(N_{1}, N_{2}\right)^{t}\left(N_{1}, N_{2}\right)\right)\right|^{\frac{1}{2}} .
$$

It is the $\left(n-n_{3}\right)$-dimensional volume of the parallelotope formed by the normal vectors of $\Sigma_{1}$ and $\Sigma_{2}$. The geometric quantity introduced is implied by the following identity for the Gramian determinant in $\left(n-n_{3}\right)$ dimensional space. This can be compared with affine-invariant formulation of Bennett \& Bez' Brascamp-Lieb inequality in terms of exterior algebra, where similar expression play comparable roles.

4.3. Polyhedral surfaces. Using the fiber representations, we are now able to deal with the general case of polyhedral surfaces - the main idea of the proof is a suitable application of CauchySchwarz on two different domains, which allows for a suitable decoupling to take place and gives rise to a much simpler linear expression.

Proposition 2. Suppose the $\Sigma_{i}$ are polyhedral surfaces and that

$$
\gamma(x, y, z) \geq \gamma_{0}
$$

whenever all three normal vectors are defined. Then, for all $f_{1} \in L^{2}\left(\Sigma_{1}\right), f_{2} \in L^{2}\left(\Sigma_{2}\right)$,

$$
\left\|f_{1} * f_{2}\right\|_{L^{2}\left(\Sigma_{3}\right)} \leq \gamma_{0}^{-\frac{3}{2}}\left\|f_{1}\right\|_{L^{2}\left(\Sigma_{1}\right)}\left\|f_{2}\right\|_{L^{2}\left(\Sigma_{2}\right)} .
$$


Proof. We assume without loss of generality that the polyhedral surfaces are made up of finitely many faces and will prove a bound uniform in the number of faces. The claim follows from the inequality

$$
\begin{aligned}
I:= & \int_{\Sigma_{3}}\left(\gamma_{0} \int_{\Gamma_{z}} \frac{\gamma(x, z-x, z)^{\frac{1}{2}}}{\gamma_{3}(x, z-x)} f_{1}(x) f_{2}(z-x) d \mathcal{H}^{n_{1}+n_{2}-n}(x)\right)^{2} d \mathcal{H}^{n_{3}}(z) \\
& \leq\left\|f_{1}\right\|_{L^{2}\left(\Sigma_{1}\right)}^{2}\left\|f_{2}\right\|_{L^{2}\left(\Sigma_{2}\right)}^{2} .
\end{aligned}
$$

which we will prove now: applying Cauchy-Schwarz inequality in the fiber $\Gamma_{z}$ yields

$$
\begin{aligned}
I \leq & \int_{\Sigma_{3}}\left(\gamma_{0} \int_{\Gamma_{z}} \frac{\gamma(x, z-x, z)^{\frac{1}{2}}}{\gamma_{3}(x, z-x)} f_{1}(x)^{2} d \mathcal{H}^{n_{1}+n_{2}-n}(x)\right) \\
& \times\left(\gamma_{0} \int_{\Gamma_{z}} \frac{\gamma\left(x^{\prime}, z-x^{\prime}, z\right)^{\frac{1}{2}}}{\gamma_{3}\left(x^{\prime}, z-x^{\prime}\right)} f_{2}\left(z-x^{\prime}\right)^{2} d \mathcal{H}^{n_{1}+n_{2}-n}\left(x^{\prime}\right)\right) d \mathcal{H}^{n_{3}}(z) .
\end{aligned}
$$

The right hand side is linear in $f_{i}^{2}$ which we exploit by localization procedure in $\Sigma_{1}$ and $\Sigma_{2}$. Take a decomposition of $\Sigma_{1}, \Sigma_{2}$

$$
\Sigma_{1}=\bigcup_{j} \Sigma_{1, j} \quad \Sigma_{2}=\bigcup_{k} \Sigma_{2, k} \quad \Sigma_{3}=\bigcup_{l}^{\cdot} \Sigma_{3, l}
$$

with the property that for each $j$ the normal vectors $\nu_{1}$ and $\nu_{2}$ are constant on the sets $\Sigma_{1, j}$ and $\left.\Sigma_{2, k}\right)$. By a further possibly countable decomposition we may also achieve that $\left(\Sigma_{1, j}+\Sigma_{2, k}\right) \cap \Sigma_{3}$ lies in a single set $\Sigma_{3, l}$. We abbreviate $f_{i, \cdot}:=f_{i} \chi_{\Sigma_{i, \cdot}}$. Using this decomposition, it remains to estimate

$$
\begin{aligned}
\int_{\Sigma_{3}}\left(\gamma_{0} \int_{\Gamma_{z}} \frac{\gamma(x, z-x, z)^{\frac{1}{2}}}{\gamma_{3}(x, z-x)} \sum_{j_{1}} f_{1, j_{2}}(x)^{2} d \mathcal{H}^{n_{1}+n_{2}-n}(x)\right) \\
\quad \times\left(\gamma_{0} \int_{\Gamma_{z}} \frac{\gamma\left(x^{\prime}, z-x^{\prime}, z\right)^{\frac{1}{2}}}{\gamma_{3}\left(x^{\prime}, z-x^{\prime}\right)} \sum_{j_{2}} f_{2, j_{2}}\left(z-x^{\prime}\right)^{2} d \mathcal{H}^{n_{1}+n_{2}-n}\left(x^{\prime}\right)\right) d \mathcal{H}^{n_{3}}(z) .
\end{aligned}
$$

Note that $f_{i, i_{1}}$ and $f_{i, i_{2}}$ have disjoint support unless $i_{1}=i_{2}$. Thus, we can expand the square and use the linearity of the integral to see that is suffices to estimate

$$
\begin{aligned}
\int_{\Sigma_{3}} & \left(\gamma_{0} \int_{\Gamma_{z}} \frac{\gamma(x, z-x, z)^{\frac{1}{2}}}{\gamma_{3}(x, z-x)} f_{1, j_{2}}(x)^{2} d \mathcal{H}^{n_{1}+n_{2}-n}(x)\right) \\
& \times\left(\gamma_{0} \int_{\Gamma_{z}} \frac{\gamma\left(x^{\prime}, z-x^{\prime}, z\right)^{\frac{1}{2}}}{\gamma_{3}\left(x^{\prime}, z-x^{\prime}\right)} f_{2, j_{2}}\left(z-x^{\prime}\right)^{2} d \mathcal{H}^{n_{1}+n_{2}-n}\left(x^{\prime}\right)\right) d \mathcal{H}^{n_{3}}(z) \\
\leq & \left\|f_{1}\right\|_{L^{2}\left(\Sigma_{1}\right)}^{2}\left\|f_{2}\right\|_{L^{2}\left(\Sigma_{1}\right)}^{2} .
\end{aligned}
$$

Note that the geometric expression $\gamma(x, z-x, z)^{\frac{1}{2}} / \gamma_{3}(x, z-x)$ is constant for every $j_{1}, j_{2}$ provided we have chosen set with sufficiently small support, because of the polyhedral natural of the surfaces and the choice of our decomposition. It is evident that $\gamma_{3}(\cdot, \cdot) \geq \gamma_{0}$ and we may thus estimate the expression from above by

$$
\begin{aligned}
J=\int_{\Sigma_{3}} & \left(\int_{\Gamma_{z}} \gamma(x, z-x, z)^{\frac{1}{2}} f_{1, j_{1}}(x)^{2} d \mathcal{H}^{n_{1}+n_{2}-n}(x)\right) \\
\times & \times\left(\int_{\Gamma_{z}} \gamma\left(x, z-x^{\prime}, z\right)^{\frac{1}{2}} f_{2, j_{2}}\left(z-x^{\prime}\right)^{2} d \mathcal{H}^{n_{1}+n_{2}-n}\left(x^{\prime}\right)\right) d \mathcal{H}^{n_{3}}(z)
\end{aligned}
$$

For every fixed $j_{1}, j_{2}$, this is now precisely the expression we had to deal with in our proof in the linear case - redoing the same steps as before yields that for every $j_{1}, j_{2}$

$$
J \leq\left\|f_{1}\right\|_{L^{2}\left(\Sigma_{1}\right)}^{2}\left\|f_{2}\right\|_{L^{2}\left(\Sigma_{1}\right)}^{2}
$$

and this concludes the proof. 
Proposition 2 implies Theorem 1. Recall that we understand the restriction $\left.f_{1} * f_{2}\right|_{\Sigma_{3}}$ in the sense of the dense embedding $C_{0}^{\infty}\left(\mathbb{R}^{3}\right) \hookrightarrow L^{2}\left(\mathbb{R}^{3}\right)$. It suffices to consider continuous functions $f_{i}$ with compact support defined on $\mathbb{R}^{n}$. But then both sides converge for $C^{1}$ hypersurfaces as the polyhedral approximation tends to the hypersurface.

\section{Proof of the Linear Brascamp-Lieb inequality}

This section gives a new proof for the linear Brascamp-Lieb inequality much in the same spirit as the proof for the convolution estimate in the linear case. Again, the proof will be stable enough to allow it being transferred to the nonlinear setting.

Proposition 3. Let $A_{i}: \mathbb{R}^{n} \rightarrow \mathbb{R}^{n_{i}}$ be linear maps with maximal rank $n_{i}$ for $1 \leq i \leq 3$, where

$$
n_{1}+n_{2}+n_{3}=2 n .
$$

Let $V_{i}$ be an orthonomal basis of the null space of $A_{i}$, let $V=\left(v_{1}, v_{2}, v_{3}\right)$ and

$$
\gamma=|\operatorname{det} V| \text {. }
$$

Then we have for all $f_{i} \in L^{2}\left(\mathbb{R}^{n_{i}}\right)$

$$
\prod_{i=1}^{3}\left|A_{i}\right|^{1 / 2} \int_{\mathbb{R}^{n}} \prod_{i=1}^{3} f_{i} \circ A_{i}(x) d x \leq \gamma^{-1 / 2} \prod\left\|f_{i}\right\|_{L^{2}\left(\mathbb{R}^{n_{i}}\right)} .
$$

Proof. Let $P$ be the parallelepiped formed by $V$. Its volume is $\gamma$. The matrix $A_{1}$ maps $P$ to a parallelepiped $P_{1}$ in $\mathbb{R}^{n_{1}}$ which is spanned be the image of the vectors of $V_{2}$ and $V_{3}$. We claim that its volume is

$$
\left|\left(W_{2} W_{3}\right)^{t}\right|^{-1}\left|A_{1}\right|=\gamma\left|A_{1}\right| \text {. }
$$

First we reduce the assertion to the case $A_{1} A_{1}^{T}=1_{\mathbb{R}^{n_{1}}}$ which we can achieve by a linear change of coordinates in $\mathbb{R}^{n_{1}}$. The volume of $P$ is the same as the volume of the parallelepiped which we obtain by orthogonally projecting the vectors $v_{2}^{a}$ and $v_{3}^{a}$ along $V_{1}$. Then we obtain $\tilde{w}_{i}^{a}$ such that $\left(V, \tilde{W}^{1}, \tilde{W}^{2}\right)$ is the dual basis to $\left(V, W^{1}, W^{2}\right)$ and the $n_{1}$ dimensional volume of the parallelepiped spanned by the columns of $\tilde{W}_{2}$ and $\tilde{W}_{3}$ is $\gamma$. We repeat this argument for the other variables and obtain

as well as

$$
\int \prod \chi_{P_{i}} \circ A_{i}(x) d x=\gamma
$$

$$
\left\|\chi_{P_{1}}\right\|_{L^{2}\left(\mathbb{R}^{n_{1}}\right)}=\left|W_{2} W_{3}\right|^{-1 / 2}\left|A_{1}\right|^{1 / 2}=\gamma^{1 / 2}\left|A_{1}\right|^{1 / 2}
$$

This implies the claimed formula with equality for characteristic functions of such parallepipeds. For general functions we proceed differently and we apply the coarea formula and Cauchy-Schwarz inequality twice:

$$
\begin{aligned}
& \prod_{i=1}^{3}\left|A_{i}\right|^{\frac{1}{2}} \int \prod_{i=1}^{3} f_{i} \circ A_{i}(x) d x \\
& \quad=\left|A_{3}\right|^{-\frac{1}{2}}\left|A_{1}\right|^{\frac{1}{2}}\left|A_{2}\right|^{\frac{1}{2}} \int_{\mathbb{R}^{n_{3}}} f_{3}(z) \int_{\left\{x: A_{3}(x)=z\right\}} f_{1}\left(A_{1} x\right) f_{2}\left(A_{2} x\right) d \mathcal{H}^{n-n_{3}} d m^{n_{3}}(z) \\
& \quad \leq\left\|f_{3}\right\|_{L^{2}\left(\mathbb{R}^{n_{3}}\right)} \int_{\mathbb{R}^{n_{3}}} \prod_{i=1}^{2}\left(\int_{\left\{x: A_{3} x=z\right\}}\left|A_{3}\right|^{\frac{1}{2}}\left|A_{i}\right|^{-1}\left|f_{i}\left(A_{i}(x)\right)\right|^{2} d \mathcal{H}^{n-n_{3}}\right) d m^{n_{3}}(z)
\end{aligned}
$$

We check the validity of the desired estimate for the special case of characteristic functions of parallepipeds $f_{i}$ for $i=1,2$ by plugging them in the right hand side expression. In this case, the integral over the fiber gives 1 and we have to integrate the very same parellepiped in $\mathbb{R}^{n_{3}}$ as above and the squares of the $L^{2}$ norms are $\left|A_{i}\right|^{-1} \times \gamma$. The argument follows for general functions by either interpreting these consideration as a determination of Gramian determinants in area and coarea formulas, or, alternatively, by approximating general continuous functions by sums of multiples of characteristic functions of parallelepipeds. 


\section{Nonlinear Brascamp-Lieb inequality: Proof of Theorem 2}

This section concludes with a proof of the Brascamp-Lieb inequality

$$
\int_{\mathbb{R}^{n}}\left(\prod_{i=1}^{3}\left|D \phi_{i}\right|\right)^{\frac{1}{2}} f_{1}\left(\phi_{1}(x)\right) f_{2}\left(\phi_{2}(x)\right) f_{3}\left(\phi_{3}(x)\right) d x \leq \sqrt{\frac{\rho}{\gamma_{0}}}\left\|f_{1}\right\|_{L^{2}\left(\mathbb{R}^{\left.n_{1}\right)}\right)}\left\|f_{2}\right\|_{L^{2}\left(\mathbb{R}^{\left.n_{2}\right)}\right)}\left\|f_{3}\right\|_{L^{2}\left(\mathbb{R}^{n_{3}}\right)} .
$$

The argument is essentially identical to our previous argument and merely phrased in a slightly different language. Previously we were dealing with the linear structure $x+y=z$ in $\mathbb{R}^{n}$ as induced by the convolution and nonlinear (but transversal) hypersurfaces. Now we are dealing with flat surfaces and a nonlinear (but transversal) fiber structure. The crucial idea is, once again, that applying Cauchy-Schwarz once on $\mathbb{R}^{n_{3}}$ and then once in the integration fiber yields a bilinear expression which, on small scales, reduces to the linear case while $L^{2}$-orthogonality allows for a reduction to small scales.

Proof of Theorem 2. It suffices to consider nonnegative functions; this implies that the integrals are defined - possibly with the value $\infty$. We start by rewriting the squared expression by the coarea formula as

$$
\left(\int_{\mathbb{R}^{n_{3}}} f_{3}(z)\left(\int_{\left\{x: \phi_{3}(x)=z\right\}}\left|D \phi_{1}\right|^{\frac{1}{2}}\left|D \phi_{2}\right|^{\frac{1}{2}}\left|D \phi_{3}\right|^{-\frac{1}{2}} f_{1}\left(\phi_{1}(x)\right) f_{2}\left(\phi_{2}(x)\right) d \mathcal{H}^{n-n_{3}}(x)\right) d m^{n_{3}}(z)\right)^{2}
$$

The next step is again $L^{2}$-duality: we apply the Cauchy-Schwarz inequality on $\mathbb{R}^{n_{3}}$ and eliminate the $f_{3}$ term entirely. We rewrite the condition $n_{1}+n_{2}+n_{3}=2 n$ as

$$
\frac{n-n_{2}}{n_{3}}+\frac{n-n_{1}}{n_{3}}=1 \quad \text { and thus } \quad\left|D \phi_{3}\right|^{-\frac{1}{2}}=\left|D \phi_{3}\right|^{-\frac{n-n_{2}}{2 n_{3}}}\left|D \phi_{3}\right|^{-\frac{n-n_{1}}{2 n_{3}}}
$$

and use the Cauchy-Schwarz inequality once more in the fiber, which results in the integral

$$
\begin{aligned}
\int_{\mathbb{R}^{n_{3}}}\left(\int_{\left\{x: \phi_{3}(x)=z\right\}}\left|D \phi_{1}\right|\left|D \phi_{3}\right|^{-\frac{n-n_{2}}{n_{3}}} f_{1}\left(\phi_{1}(x)\right)^{2} d \mathcal{H}^{n-n_{3}}(x)\right) \\
\times\left(\int_{\left\{x: \phi_{3}(x)=z\right\}}\left|D \phi_{2}\right|\left|D \phi_{3}\right|^{-\frac{n-n_{1}}{n_{3}}} f_{2}\left(\phi_{2}(x)\right)^{2} d \mathcal{H}^{n-n_{3}}(x)\right) d m^{n_{3}}(z) .
\end{aligned}
$$

It is crucial to be aware of the arising dimensions: the total integral is an integral in dimension $2\left(n-n_{3}\right)+n_{3}=n_{1}+n_{2}$, and we want to bound it in terms of the square of the $L^{2}$ norms, which again is a related to an integral over a set of dimension $n_{1}+n_{2}$. The transversality condition implies that there is a bijective mapping between the sets $\Sigma_{1} \times \Sigma_{2}$ and

$$
\left\{(x, y) \in \mathbb{R}^{n} \times \mathbb{R}^{n}: \phi_{3}(x)=\phi_{3}(y)\right\} .
$$

Instead of constructing and working with this map directly we choose a more geometric and less technical approach: it suffices again to verify the estimate for functions $f_{1}$ and $f_{2}$ supported on small parallelepipeds (which is implicitly a construction of the map between the two spaces). Indeed, if we can prove the inequality for characteristic functions

$$
\left(f_{1}, f_{2}\right)=\left(\chi_{E}, \chi_{F}\right)
$$

for small parallelepipeds $(E, F) \subset \mathbb{R}^{n_{1}} \times \mathbb{R}^{n_{2}}$, the entire inequality follows from the bilinearity of the expression and the $L^{2}$-orthogonality of functions with disjoint support by mere addition. As in the case of the convolution, it suffices to consider piecewise linear maps $\phi_{i}$. Now let $x, y \in \mathbb{R}^{n}$ such that $\phi_{3}(x)=\phi_{3}(y)$. We may restrict ourselves to linear maps $A_{1}=\phi_{1}$ and $A_{2}=\phi_{2}$ as well as $\phi_{3}=A_{3}^{x}$ and $\phi_{3}=A_{3}^{y}$ near $x$ resp. $y$. The two linear maps will differ in general. There is no harm in applying a rotation $O$ at $x$. Hence we may assume that the null spaces of $A_{3}^{x}$ and $A_{3}^{y}$ are the same. We proceed as in the linear situation for which all quantities have been explicitely computed, with a linear map $A_{3}$ defined by the null space, by $A_{3}=A_{3}^{x}$ on the null space of $A_{1}$ and by $A_{3}=A_{3}^{y}$ on the null space of $A_{2}$.

The only difference to the previous case concerns the third map. The Gramian determinant is given by the volume of the parallelepiped spanned by image of $V_{1}$ under the map $A_{3}^{x}$ and $V_{2}$ under 
the map $A_{3}^{y}$, respectively. This volume is certainly biggest if these maps induce an orthogonal image, in which case it is bounded by the product of the $\left(n-n_{1}\right)$ dimensional volume of the image of $V^{1}$ under $A_{3}^{x}$ and the $\left(n-n_{2}\right)$ dimensional volume of the image of $V^{2}$ under $A_{3}^{y}$. Comparison with the linear case shows that this factor is controlled by the product of

$$
\rho_{1}(x)=\left|D \phi_{3}(x)\right|^{-\frac{n-n_{2}}{n_{3}}} \prod_{j=n-n_{2}+1}^{n_{3}} \sigma_{j} \quad \text { and } \quad \rho_{2}(y)=\left|D \phi_{3}(x)\right|^{-\frac{n-n_{1}}{n_{3}}} \prod_{j=n-n_{1}+1}^{n_{3}} \sigma_{j} .
$$

Acknowledgments. We are grateful to Sebastian Herr for valuable discussions. The second author was supported by a Hausdorff scholarship of the Bonn International Graduate School and SFB 1060 of the DFG.

\section{REFERENCES}

[1] I. Bejenaru, S. Herr. Convolutions of singular measures and applications to the Zakharov system, Journal of Functional Analysis (2011) Vol. 261, No. 2, pp. 478-506.

[2] I. Bejenaru, S. Herr, D. Tataru. A convolution estimate for two-dimensional hypersurfaces. Rev. Mat. Iberoam. 26 (2010), no. $2,707-728$.

[3] J. Bennett, N. Bez. Some nonlinear Brascamp-Lieb inequalities and applications to harmonic analysis. J. Funct. Anal. 259 (2010), no. 10, 2520-2556.

[4] J. Bennett, A. Carbery, J. Wright. A non-linear generalisation of the Loomis-Whitney inequality and applications. Math. Res. Lett. 12 (2005), no. 4, 443-457.

[5] J. Bourgain, Refinements of Strichartz' inequality and applications to 2D-NLS with critical nonlinearity, Internat. Math. Res. Notices 5 (1998), 253-283.

[6] L. Loomis, H. Whitney, An inequality related to the isoperimetric inequality, Bull. Amer. Math. Soc. 55 (1949), 961-962 .

Mathematisches Institut, Universität Bonn, Endenicher Allee 60, 53115 Bonn, Germany

E-mail address: koch@math.uni-bonn.de

Department of Mathematics, Yale University, 10 Hillhouse Avenue, CT 06511, USA

E-mail address: stefan.steinerberger@yale.edu 